\title{
Water Content Measurement in Expansive Soils Using the Neutron Probe
}

\begin{abstract}
Capacitance-type methods for measuring soil water content are known to be unreliable in expansive soils, as cracking disrupts the intimate contact between the soil and the measuring device. The neutron probe, which infers water content from the thermalisation of a cloud of neutrons, is potentially less affected by cracking. The effect of cracking on neutron probe measurements was investigated by a series of numerical simulations using an axisymmetric finite element model based on seven-group neutron-diffusion theory. The simulations employed a consistent soil cracking model based on Maryland clay, in which crack volumes are determined from the changes in void ratio in the shrinking bulk soil. The results show that the presence of cracks in a clay soil affects the inferred water content and that measurements affected by air-filled cracking under-predict not only the water content in the uncracked soil peds but also the average water content in the larger cracked soil mass. The reason for this underprediction is understood by considering the spatial distribution of the thermalised neutrons in the cracked and uncracked soils. The fast neutrons emitted from the source are seen to diffuse preferentially along air-filled cracks, traveling a large distance from the detector before they become thermalised, thus reducing their likelihood of being back-scattered to the detector where they can be counted. The proximity of the first crack to the probe in the ground also affects the measurement. Water-filled cracks are seen to have the opposite (but lesser) effect to air-filled cracks. A comparison of a simple uniform width crack model to a more realistic model in which crack width varies with changing water content shows that the model is sensitive to crack distribution and that the linear calibration expressions that are typically employed for neutron probes are likely to be unreliable in cracked clay soils.
\end{abstract}

KEYWORDS: neutron probe, finite element model, cracks

\section{Introduction}

There is a variety of methods available for determining in situ soil moisture content. Unfortunately the use of most of these in expansive clay soils is complicated by the formation of desiccation cracks that tend to occur preferentially between the soil and the embedded measurement infrastructure. Figure 1, for example, shows the root zone $(0-300 \mathrm{~mm})$ soil moisture recorded by a capacitance-type device at an expansive clay site in the Hunter Valley, Australia. The development of cracks in and around the embedded metal rods of the device has seriously disrupted its ability to record the soil moisture with consistent accuracy over time, producing observations of soil moisture that jump between unrealistic values depending on whether the cracking, which has formed around the waveguide rods, is open or closed or filled with air or water.

The neutron moisture meter, or neutron probe, offers an alternative to the capacitance-type soil moisture devices (see Li et al. (2003a, 2003b)). The neutron probe infers the overall water content of a volume of soil by measuring the extent of thermalisation of a diffuse cloud of neutrons and thereby has the potential to determine the overall water content equally well in both cracked and uncracked soils. The neutron probe employs a source of fast, high energy neutrons $\left({ }^{241} \mathrm{Am}-\mathrm{Be}\right)$ in combination with a slow (thermal) neutron detector, in a device which can be lowered down a pre-

Manuscript received November 1, 2009; accepted for publication May 10, 2010; published online February 2011.

${ }^{1}$ Associate Professor, Priority Research Centre for Geotechnical and Materials Modelling, The School of Engineering, The Univ. of Newcastle, Callaghan, Newcastle 2308, Australia, e-mail: Stephen.Fityus@newcastle.edu.au

${ }^{2}$ Senior Fellow, The School of Engineering, The Univ. of Newcastle, Callaghan, Newcastle 2308, Australia, e-mail: Tony.Wells@newcastle.edu.au

${ }^{3}$ Professor, Dept. of Engineering Mechanics, Hohai University, Nanjing 210098, China, e-mail: wh670@hhu.edu.cn drilled borehole to determine soil moistures at various depths (Fig. 2). Fast neutrons emitted from the source diffuse into the surrounding soil and collide with the atomic nuclei of the soil, transferring energy from the neutrons to the nuclei and slowing the neutrons. In collisions with large, heavy nuclei, the neutrons rebound without being significantly slowed. However when neutrons collide with hydrogen atoms, which have an almost identical mass to the neutron, the neutrons are slowed down more effectively, and after a small number of collisions, the energy of the neutrons is reduced to the point (known as thermalisation) at which the neutron probe sensor can detect them. In soils with low water content (and hence relatively low volumetric concentration of hydrogen), a large low density thermalised neutron cloud is formed around the probe, which then records a low neutron count. As soil moisture increases, the thermal neutron cloud shrinks and becomes denser, which results in an increase in the detection of back-scattered thermal neutrons.

The manner in which the neutron probe operates suggests that

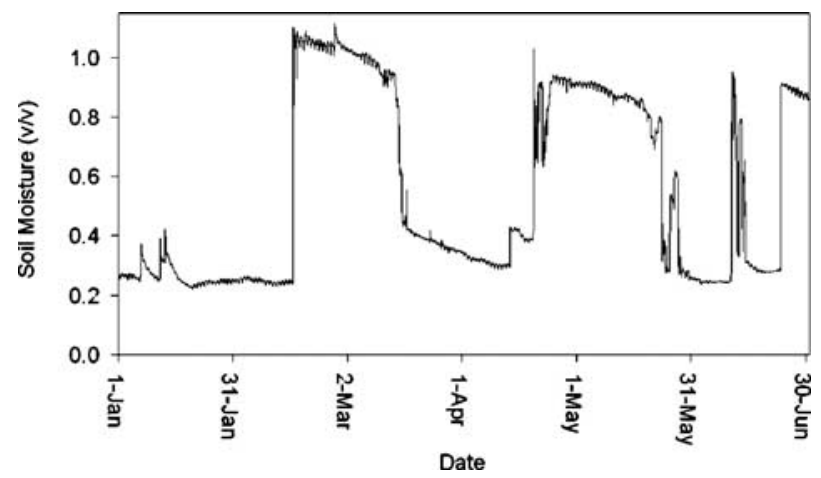

FIG. 1-Soil moisture data measured in situ using a capacitance probe in an expansive soil in the Hunter Valley, Australia. 


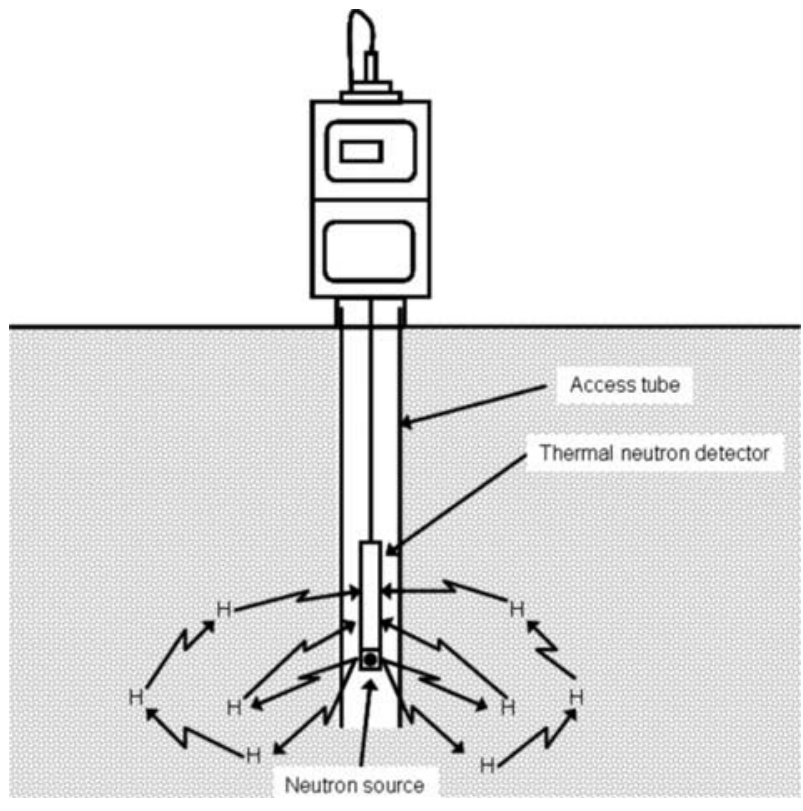

FIG. 2 -Schematic representation of the neutron probe.

the reported neutron count is simply a function of the total water content of the surrounding soil volume (whether cracked or not) and hence would appear to be less prone to the soil cracking induced errors shown above for capacitance probes. Nevertheless it has been shown that gaps between the access tube and the soil profile can influence the response of the neutron probe (Amoozegar et al. 1989; Li et al. 2003a). This indicates that while the neutron probe may function in a cracked clay environment the readings generated by the probe may to some extent be influenced by the presence of cracks in the surrounding soil media. Greacen and Hignett (1979), for example, observed biases brought about by bulk density differences and non-representivity of the access tube when using the neutron probe in clay soils. Jarvis and Leeds-Harrison (1987) also point to problems with cracking around the access tube in clay soils as being a possible source of error. A number of studies (for example, Greacen and Hignett (1979), Jayawardane et al. (1984), and Corbeels et al. (1999)) show that correcting for textural and bulk density changes can markedly improve neutron probe calibration; however, as pointed out by Jayawardane, individual bulk density-moisture relationships for each soil layer are required for the best results. Grismer et al. (1995) on the other hand notes that bulk density corrections do not necessarily improve the standard errors of water content measurement and suggests that soil sampling for calibration within the access tube hole reduces measurement errors. The difficulty of obtaining representative soil samples for calibration purposes in cracking soils has been highlighted by a number of researchers (Jarvis and Leeds-Harrison 1987; Corbeels et al., 1999), who concluded that using undisturbed core and clod sampling gives the best chance of success in calibrating the neutron probe in clay soils.

The difficulties inherent in the field calibration of the neutron probe in cracking clay soils highlight the need to develop theoretical models that aid in understanding how the probe functions in these heterogeneous environments. To determine the manner and extent to which cracks in the surrounding soil influence the output of the neutron probe, a finite element model of the probe operation in a cracked clay environment is constructed with the aim of evaluating the impact of air- and water-filled desiccation cracks on neu- tron probe readings (Wells and Fityus 2007). Using clay soil behaviour models that capture the changes in volume and dry density in real desiccated soils, the influence of crack size, density, and orientation on neutron counts is determined for a range of soil moistures.

\section{Numerical Model of the Neutron Probe Test}

The neutron probe measurement of soil water content is governed by the scattering, absorption and slowdown of fast neutrons as they undergo collisions with atomic nuclei within the soil medium. The stabilized neutron flux consists of neutrons distributed across an energy spectrum ranging from $4.5 \mathrm{MeV}$ (the initial fast neutrons generated by the probe source) to less than $2 \mathrm{eV}$ (the thermalised state). Ølgaard (1965) proposed the use of a neutron-diffusion model in which the neutrons were grouped into three energy bands and showed that it can produce results in reasonable agreement with experimental measurements. Similar models were also used been used by Haahr and Ølgaard (1965), Greacen and Schrale (1976), Elder and Rasmussen (1994), and Iliffe (1982). In many situations, a seven-group diffusion model has been shown to provide a good estimate of the thermal neutron flux (e.g., Ritchie and Wilson (1984)).

While neutron fluxes can be calculated from analytical solutions of the three-group diffusion equations in an infinite domain, boundary effects and non-uniform multi-domains are difficult to incorporate into such an approach. To overcome this, an improved sevengroup diffusion model has been developed in which the system of diffusion equations is solved in multiple spatial domains using the finite element method ( $\mathrm{Li}$ et al. 2003a). This allows for a realistic consideration of effective boundary conditions and a better assessment of the impacts of various factors such as the presence of an access tubes and the proximity of free surfaces and water tables. It also facilitates simulation of laboratory-based measurements in drums. The numerical model solves the following system of diffusion equations in defined spatial domains (Li et al. 2003a):

$$
\left\{\begin{array}{c}
-D_{1} \nabla^{2} \phi_{1}+\Sigma_{1}^{s l} \phi_{1}+\Sigma_{1}^{a} \phi_{1}=S \quad E_{1} \geq E>E_{2} \\
-D_{2} \nabla^{2} \phi_{2}-\Sigma_{1}^{s l} \phi_{1}+\Sigma_{2}^{s l} \phi_{2}+\Sigma_{2}^{a} \phi_{2}=0 \quad E_{2} \geq E>E_{3} \\
-D_{3} \nabla^{2} \phi_{3}-\Sigma_{2}^{s l} \phi_{2}+\Sigma_{3}^{s l} \phi_{3}+\Sigma_{3}^{a} \phi_{3}=0 \quad E_{3} \geq E>E_{4} \\
\cdots \cdots \\
\cdots \cdots \\
-D_{7} \nabla^{2} \phi_{7}-\Sigma_{6}^{s l} \phi_{6}+\Sigma_{7}^{a} \phi_{7}=0 \quad E_{7} \geq E
\end{array}\right.
$$

where:

$\phi_{i}$ represents the neutron flux at the energy level of $E_{i} \geq E$ $\geq E_{i+1}$,

$D_{i}=$ diffusion coefficient for neutrons in the energy band $E_{i}$ $\geq E \geq E_{i+1}$,

$\Sigma^{s l}{ }_{i}$ and $\Sigma^{a}{ }_{i}=$ slowdown cross section and the absorption cross section (energy band $E_{i} \geq E \geq E_{i+1}$ ), respectively, and

$S=$ source intensity, which is nonzero only in the source domain.

The diffusion coefficient $D_{i}$ and the cross sections $\Sigma^{s l}{ }_{i}$ and $\Sigma^{a}{ }_{i}$ are determined by the chemical composition of the soil and also depend on the neutron energy level ( $\mathrm{Li}$ et al. 2003a). The absorption of fast neutrons in soils is minor and can be neglected in a numerical analysis with little loss of accuracy. Details of the determination of $\Sigma^{s l}{ }_{\mathrm{i}}, \Sigma^{a}{ }_{\mathrm{i}}$, and $D_{i}$ and the grouping of energy levels for the americium-beryllium $\left({ }^{241} \mathrm{Am}-\mathrm{Be}\right)$ source neutron probe can be found in Li et al. (2003a). Once the neutron fluxes are computed, the neutron counts detected per unit time can be estimated by the following integration over the spatial domain of the sensor: 
TABLE 1-Chemical composition in soils, expressed in percentage of oven-dry weight (Greacen and Schrale 1976).

\begin{tabular}{lcccccccccccccccc}
\hline Soil & $\mathrm{Si}$ & $\mathrm{Na}$ & $\mathrm{Al}$ & $\mathrm{H}$ & $\mathrm{Fe}$ & $\mathrm{O}$ & $\mathrm{Ti}$ & $\mathrm{P}$ & $\mathrm{Mn}$ & $\mathrm{N}$ & $\mathrm{Ca}$ & $\mathrm{C}$ & $\mathrm{Mg}$ & $\mathrm{B}$ & $\mathrm{K}$ & $\mathrm{Cl}$ \\
\hline Long Flat clay & 27.53 & 0.60 & 8.90 & 1.14 & 4.80 & 49.40 & 0.498 & 0.073 & 0.038 & 0.40 & 0.58 & 4.00 & 0.74 & 0.006 & 1.44 & 0.017 \\
Konetta soil & 15.96 & 0.077 & 2.392 & 0.21 & 1.614 & 49.60 & 0.142 & 0.01 & 0.023 & 0.000 & 21.79 & 6.74 & 0.866 & 0.002 & 0.577 & 0.018 \\
\hline
\end{tabular}

$$
C=\int_{v} \phi_{\mathrm{th}} \sum_{a, D} d v
$$

where:

$C=$ neutron count,

$\phi_{\mathrm{th}}=$ computed thermal neutron flux, and

$\Sigma_{a, D}$ represents the thermal absorption cross-section of the neutron sensor.

Experimental results suggest a nearly linear relationship between the neutron count and volumetric water content in soil media (e.g., Ølgaard (1965)). Therefore a linear calibration curve is usually assumed for the neutron probe

$$
\theta=a C_{r}+b
$$

where:

$\theta=V_{w} / V$ represents the volumetric water content in a soil volume $V$ and

$C_{r}=C / C_{w}=$ neutron count ratio of the measured neutron count $C$ in the soil over the measured neutron count $C_{w}$ in water.

The neutron count ratio is used instead of neutron count to account for changes in source strength, which varies from one probe to another and also in the same probe over time due to the radioactive decay. For a specific soil the constants $a$ and $b$ have to be determined in a calibration procedure before using the probe for reliable routine measurements. Note that in all of the simulations that follow, the probe is modelled according to the details for a CPN503 model neutron probe (CPN International, Inc.).

\section{Verification of Numerical Model in Clay Soils without Cracking}

To verify the numerical model, drum calibration tests on two nonexpansive soils presented by Greacen and Schrale (1976) were modelled using commercially available finite element software. The coefficients in Eq 1 are determined for the chemical components of the Long Flat clay and Konetta soil as listed in Table 1, cited from Greacen and Schrale (1976). The drum employed in the study was $68 \mathrm{~cm}$ in diameter by $80 \mathrm{~cm}$ high, filled with soil, with an aluminum access tube installed in the centre. It was modelled as a two-dimensional cylindrically symmetrical problem. A constant dry soil density, corresponding to the average value of all of the tested samples, was assumed in the determination of the diffusion equation coefficients $\left(\rho_{d}=0.922 \mathrm{~g} / \mathrm{cm}^{3}\right.$ (Long Flat clay) and $\rho_{d}$ $=1.31 \mathrm{~g} / \mathrm{cm}^{3}$ (Konetta soil)).

The predicted neutron count ratios $C_{r}$ as a function of volumetric water content $\theta$ are presented in comparison with the measured data in Fig. 3. It can be seen that the model predictions based on the average dry density lie within the scatter of the measured data, as expected. In the range of $\theta=0.1-0.4$, a roughly linear relation between $C_{r}$ and $\theta$ is predicted. This validates the practice of assuming a linear calibration for a specific soil based on parameters $a$ and $b$ given by Eq 3 .

The results indicate that use of the access tube, which is assumed full of air, significantly influences the neutron probe readings. In Fig. 3(a), the dash-dotted curve is obtained on the assumption that the neutron probe is buried in the soil without using an access tube. The use of an access tube appears to lead to some neutron leakage, resulting in a much lower neutron count. As a result, the calibration parameters obtained using Eq 3 to fit the experimental data may lead to an unrealistic negative soil moisture value being predicted at low neutron counts. The model results in which the effects of the access tube are included (solid line in Fig. 3(a) and $3(b))$ predict a slight turn in the $C_{r}-\theta$ curve at around $\theta=5 \%$, which indicates that a linear calibration is only applicable for water contents greater than this value. When using the neutron probe for the measurement of water content, a calibration procedure is always required. The results in the previous section demonstrate that this task can now be reliably achieved through numerical modelling, which allows a variety of factors that may affect the calibration to be readily assessed.

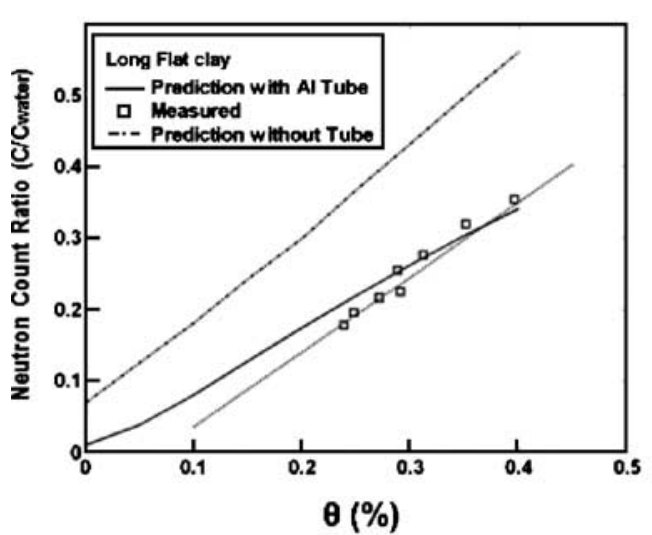

(a)

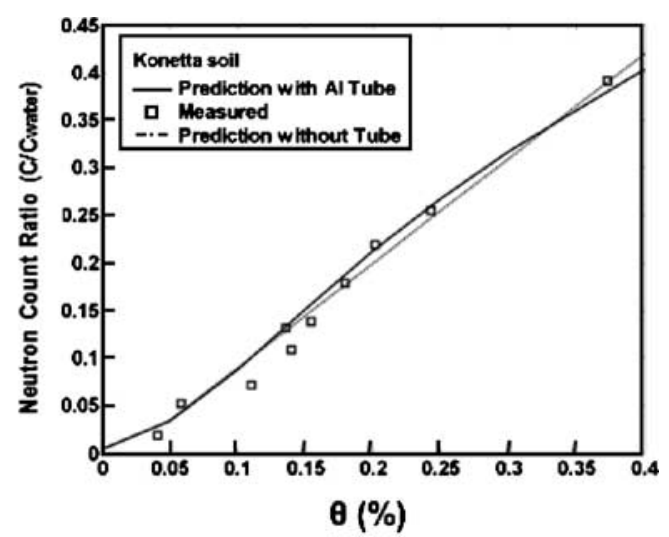

(b)

FIG. 3-Model predictions and test measurement for (a) Long Flat clay and (b) Konetta soil. 


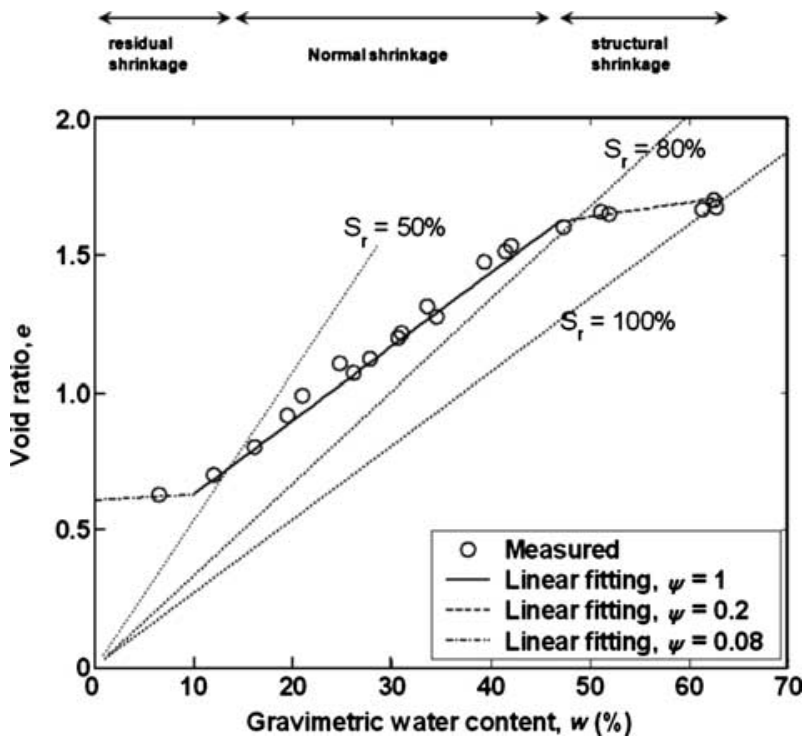

FIG. 4-A typical shrink-swell curve for expansive soil (Tripathy et al. 2002) and linear fitting with $\mathrm{Eq} 5$.

\section{A Simple Cracking Expansive Soil Behaviour Model}

Cracking is a common macro-structure feature associated with the drying and wetting of expansive soils and an unavoidable consequence of isotropic shrinkage in extensive soil bodies. Under repeated drying/wetting cycles, stable micro- and macro-structures generally develop in the soil with stable material properties (Kodikara et al. 2002). As an expansive soil shrinks and cracks, its water content reduces and the dry density of the uncracked soil elements increases significantly. As this affects the concentration of atomic nuclei in the soil, it must be accounted for in the model. There is a corresponding decrease in the bulk density, which can be greater or less, depending upon whether the bulk density of the whole cracked soil or the soil peds is considered.

The shrinking and swelling behaviour of expansive soils has been the subject of much research (e.g., Hanafy (1991)). Haines (1923) observed that shrinkage of natural soils in a progressive drying process consists of three distinct phases, namely, structural shrinkage, normal shrinkage, and residual shrinkage, as illustrated in Fig. 4. In the structural shrinkage phase, the larger interconnected pores in a soil are drained with a corresponding soil volume decrease less than the volume of water lost. During the normal shrinkage phase, soil volume decrease is approximately equal to the volume of water lost: Pore size reduces as water is lost, and the soil remains at, or close to, saturated levels (Fityus and Buzzi 2009). The lower limit of the water content for the normal shrinkage phase is known as the shrinkage limit. Further drying of the soil below this shrinkage limit leads to air-entry (desaturation), and there is little volume change as water is lost during the residual shrinkage phase. In Fig. 4, these three phases are illustrated in the results of cyclic drying/wetting tests on a compacted expansive soil (Tripathy et al. 2002). Two important behaviours are apparent. (i) Normal shrinkage dominates the wetting/drying behaviour, corresponding to gravimetric water contents from $\sim 10 \%$ to $45 \%$, respectively. (ii) The linear normal shrinkage section of the swellshrink curve is almost parallel to the saturation line.

In the normal shrinkage phase, a linear relation between the void ratio $e$ and the gravimetric water content $w$ has been experimentally verified (Fityus and Buzzi 2009). It can be assumed that the volume change of the soil element $V$ is proportional to the volume of the water $V_{w}$, lost or gained

$$
d V=\psi d V_{w}
$$

where:

$\psi=$ proportionality constant $\leq 1$.

Here, the limiting situation of $\psi=1$ corresponds to an equality between the volume of soil shrinkage and the volume of water lost during drying. By assuming that $\psi$ is a constant throughout the equilibrium cycles of drying and wetting, the following linear relation can be derived from consistency conditions:

$$
e-e_{0}=\psi G_{s}\left(w-w_{0}\right)
$$

where:

$w_{\mathrm{o}}$ denotes the gravimetric water content,

$e_{o}$ denotes the void ratio at the shrinkage limit, and

$G_{s}=$ specific gravity of soil solids.

Equation 5 is consistent with the experimental observation that a linear relationship exists between $e$ and $w$, and it implies that the saturation line in the $e-w$ plane has a gradient of $G_{s}$. With respect to Fig. 4 and Eq 5, the assumption of constant $\psi$ can further be extended to the structural shrinkage phase and residual shrinkage phase. These two phases can also be approximated with linear $e-w$ relations, only with much smaller $\psi$ values.

The variation of the dry density, $\rho_{d}$, with respect to $w$ can be obtained from

$$
\rho_{d}=\frac{G_{s} \rho_{w}}{1+e}
$$

Referring to Eq 5 it is clear that $\rho_{d}$ depends nonlinearly on $w$. The volumetric and the gravimetric water content $\theta$ and $w$ are related nonlinearly through

$$
\theta=\left(\rho_{d} / \rho_{w}\right) w
$$

A linear relationship however between $\rho_{d}$ and $\theta$ can be derived

$$
\frac{\rho_{d}}{\rho_{d 0}}=\frac{1-\psi \theta}{1-\psi \theta_{0}}
$$

\section{Application of the Soil Model to the Maryland Expansive Clay Soil}

In this work, the soil behaviour model is applied to the Maryland expansive clay soil (Fityus et al. 2004) for use in the neutron probe numerical model. The expansive soils at the Maryland test site consist of residual silty clays from medium to high plasticity to depths of $\sim 1.8 \mathrm{~m}$. The geotechnical profile for the soil at the site, which varies gradually over the depth, can be found in Fityus et al. (2004). In the analyses of this paper, the soil is treated as a single layer and soil properties are averaged over the layer. The numerical approach described here however can readily be used to model stratified profiles. Some properties of this soil relevant for this study are listed in Table 2, where $I_{p}$ is the plasticity index and LS denotes the linear shrinkage. The chemical composition of Maryland clay is listed in Table 3 .

It is observed that although the water content of the soil at Maryland test site varies seasonally, it generally remains within the range of the normal shrinkage phase. In fact, the residual and structural shrinkage phases are narrow and outside the usual range of water 
TABLE 2-Some basic properties of Maryland soil.

\begin{tabular}{lcccccc}
\hline & \multicolumn{5}{c}{ Property } \\
\cline { 2 - 6 } & $I_{p}, \%$ & $L S, \%$ & $G_{s}$ & $w_{0}, \%$ & $e_{0}$ \\
\hline Value & 40 & 15 & 2.68 & 16 & 0.429 \\
\hline
\end{tabular}

content change observed in the field. Hence in this study, the soil volume change is assumed to occur predominantly in the normal shrinkage phase. The field soil profile is assumed to consist of blocks of soil (peds), which are separated by cracks that form a network of air-filled open spaces between the peds. The cracks open up in dry weather and close up when the water content is greater than a critical value (the field capacity). They may also be waterfilled in the short term (for example, after heavy rain). Within the peds, the soil is single-structured with micro-pores only so that there is no structural shrinkage and normal shrinkage begins immediately as water is lost from the field capacity water content. This behaviour has been verified in the laboratory where the normal shrinkage regime for remoulded Maryland soil was observed to operate between $w_{\mathrm{o}}=16 \%$ and $w_{1}=44 \%$. The relations of Eqs 4-8 applied to Maryland clay are plotted in Fig. 5.

\section{Implementation of the Numerical Model}

To investigate the role of cracking on the neutron probe output a numerical model of the neutron probe arrangement was constructed within a three-dimensional (3D) axisymmetric finite element framework using a commercially available software package. The basic geometry of the domain used in the finite element model is illustrated in Fig. 6. In this study the neutron access shaft was 47 $\mathrm{mm}$ inside diameter by $1.7 \mathrm{~m}$ long, and the neutron source was situated $1.5 \mathrm{~m}$ below ground level with the sensor mounted $30 \mathrm{~mm}$ above the source. Due to the axisymmetric nature of the model, the cracks take the form of cylindrical shells (for vertical cracks) and planar disks (for horizontal cracks). The dimensions of the total soil volume domain ( $3 \mathrm{~m}$ deep with a radius of $2.5 \mathrm{~m}$ ) were large enough to prevent significant neutron flux leaving through the bottom and outer radial surfaces of the soil volume.

In all but one analysis, the soil dry density and crack density were determined using the soil behaviour model described above. Diffusional, collisional, and absorptive coefficients for the soils employed in Eq 1 were calculated using the approach outlined in our previous work ( $\mathrm{Li}$ et al. 2003a) and the elemental composition of silty clay soil found at a research site located $10 \mathrm{~km}$ West of the city of Newcastle, Australia (see Table 4 in Li et al. (2003a)). Airfilled cracks were assumed to contain air at $20^{\circ} \mathrm{C}$ and $50 \%$ relative humidity.

\section{Results}

\section{The Effect of Dry Density Changes on Neutron Probe Readings}

In the first analysis, consideration was given to the effect of shrinkage on soil density, and its significance for measurements made using the neutron probe. To see this effect clearly, the first analysis was conducted by modelling uncracked soils. The variation of soil dry density with the water content was computed according to Eqs 5 and 6 . With an assumed value of $\psi=1$ for the normal shrinkage phase, the dry density of the soil varied between $\rho_{d o}=1.876$ and $\rho_{d 1}=1.300$ in the range of $w_{\mathrm{o}} \leq w \leq w_{1}$. Correspondingly, the volumetric water content, $\theta$, varied in the range of 30.0-54.1\% (Fig. 5). For the purposes of comparison, neutron counts were also computed for the same soil with a constant dry density averaged over the whole range of water content change: $\bar{\rho}_{d}=1.407$. In this case, the volumetric water content, $\theta$, varied between $23.5 \%$ and $61.9 \%$.

The neutron counts are presented against the volumetric water content $\theta$ in Fig. 7. It can be seen that whilst the relationships are slightly curved, a linear expression provides a good approximation for $C_{r}-\theta$ data. The influence of the density variation can be quantified by the difference between the relevant values for the calibration parameters. In the case where constant density is assumed, the $C_{r}-\theta$ data can be fitted by Eq 3 with parameters $a=0.1393$ and $b$ $=0.054$. When the density variation is taken into account, the calibration parameters become $a=0.1174$ and $b=0.066$. This difference is small but significant.

\section{The Effect of Air-Filled Vertical Cracking}

To explore the basic effect of vertical air-filled cracks on the neutron probe readings, a series of analyses was performed on cracked soils at different water contents with crack widths of 5, 7.5, and 10 $\mathrm{mm}$. To simplify the considerations here, it was assumed that the soil had a $10 \%$ void fraction $(\mathrm{v} / \mathrm{v})$ at all water contents. While this is not consistent with the cracking soil model described above, it allows the effect of simple air-filled cracks to be considered more directly. Accordingly, crack spacing was calculated to ensure that the constant average void fraction was maintained, requiring that the closest cracks be positioned a distance of 95, 143, and $190 \mathrm{~mm}$ from the probe axis, respectively. The results are shown in Fig. 8.

It is clear that the presence of cracking in the soil leads to a significant decrease in the neutron count. For a constant void fraction, the greatest decrease in the neutron count was predicted for the case when finer, more numerous cracks were present. For particularly coarse $(10 \mathrm{~mm})$ cracking, the discrepancy between cracked and non-cracked soil neutron count was more pronounced at drier conditions; however this was not observed for finer cracked soils.

The presence of vertical air-filled cracks leads in all cases to an under-prediction of the soil moisture. This is so both in bulk soil terms and in terms of the soil moisture present in the desiccated soil peds. As revealed in Fig. 9, which compares the measurement predictions with the bulk soil and soil ped water contents, the underestimation can be as high as $0.08 \mathrm{v} / \mathrm{v}$ for $10 \mathrm{~mm}$ cracking.

Whilst it is expected that the neutron probe should underesti-

TABLE 3-Chemical composition of Maryland clay soil, expressed in percentage of oven-dry weight (Li et al. 2003 a).

\begin{tabular}{|c|c|c|c|c|c|c|c|c|c|c|c|c|c|c|c|c|}
\hline Soil & $\mathrm{Si}$ & $\mathrm{Na}$ & $\mathrm{Al}$ & $\mathrm{H}$ & $\mathrm{Fe}$ & $\mathrm{O}$ & $\mathrm{Ti}$ & $\mathrm{P}$ & $\mathrm{Mn}$ & $\mathrm{N}$ & $\mathrm{Ca}$ & $\mathrm{C}$ & $\mathrm{Mg}$ & B & K & $\mathrm{Cl}$ \\
\hline Maryland soil & 30.20 & 0.56 & 8.64 & 0.38 & 4.10 & 52.51 & 0.37 & 0.013 & 0.005 & 0.12 & 0.03 & 0.41 & 0.55 & 0.005 & 2.06 & 0.046 \\
\hline
\end{tabular}



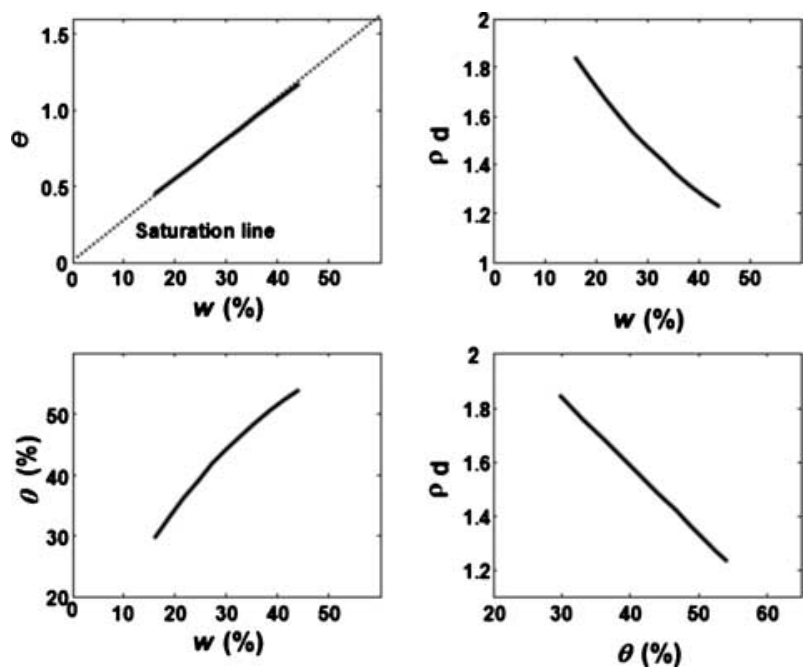

FIG. 5-A set of consistent soil property relations obtained by applying the normal shrinkage soil model to cracking Maryland clay: $e=$ void ratio; $w$ $=$ gravimetric water content; $\theta=$ volumetric moisture content; and $\rho_{d}=d r y$ density (Huang and Fityus 2008).

mate the water content in the soil peds, it might also be reasonable to expect that it should accurately predict the water content in the bulk cracked soil. To understand why it does not, it is necessary to look at the distribution of thermalised neutrons throughout the soil in the model, (for example as shown in Fig. 10 for $10 \mathrm{~mm}$ vertical air-filled cracks). From Fig. 10, it is apparent that in the case of vertical air-filled cracks, neutrons are redistributed vertically up and down the soil profile within the cracks. This is due to fact that air has a low molecular density, and consequently air-filled cracks are quite transparent to neutrons of all energies and act as preferential pathways for their redistribution. This allows the neutrons to travel further away from the sensor before they are thermalised, effectively lowering the probability of thermalised neutrons returning to the probe sensor. In the presence of cracks the neutron cloud is "smeared" vertically, with the net effect being a decrease in neutron population in the region of the probe sensor.

\section{The Effect of Crack Proximity to the Neutron Probe}

To further illustrate the redistribution effect, modelling was conducted in which the location of the crack closest to the probe was

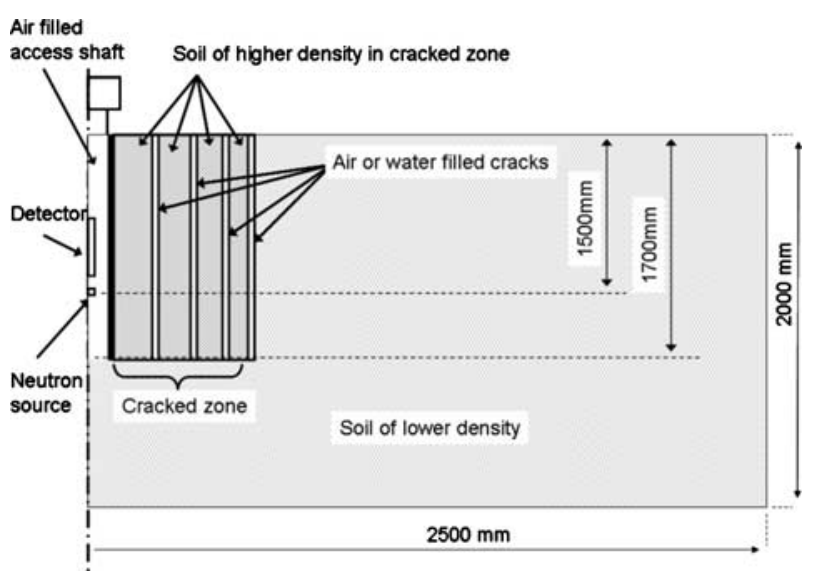

FIG. 6-The general axisymmetric layout used for the numerical analysis. (Note the number and orientation of cracks was varied.)

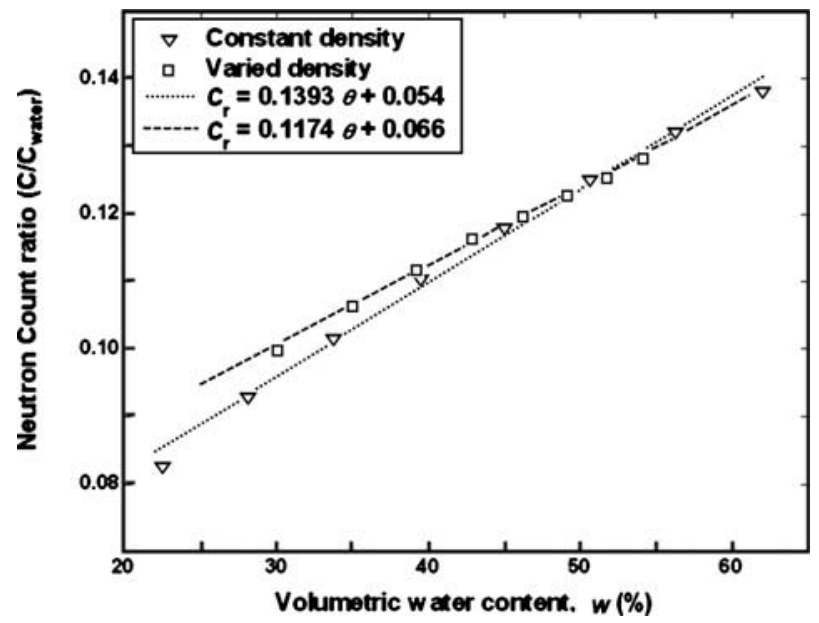

FIG. 7-Density effect on neutron count ratio for Maryland soil.

altered to embed it further into the high density neutron cloud adjacent to the neutron source (Fig. 11). For both dry and high moisture soils, it is clear that the closer nearest crack is to the probe, the greater the effect on the neutron probe reading. Conversely, as the inner edge of the cracked zone is moved away from the probe the effect is lessened, particularly at higher moisture values. At higher moisture levels the neutron cloud is naturally contracted (as discussed above) and hence soil cracks at a given distance from the probe are not as deeply embedded in the cloud thereby reducing their influence on the neutron count.

\section{The Effect of Water-Filled Vertical Cracking}

Model predictions clearly indicate that air-filled cracks act to distort and ultimately disperse neutrons from the neutron probe resulting in a reduction in the neutron count. Water-filled cracks however would be expected to behave differently as water is a very efficient absorber and thermaliser of neutrons and it has a density comparable with that of the surrounding soil. Figure 12 shows the effect of the presence of water-filled $7.5 \mathrm{~mm}$ vertical cracks on the response of the neutron probe for cracked soils with an overall porosity of $10 \%$.

It is evident from the trends shown in Fig. 12 that the influence of water-filled cracks on the response of the neutron probe is complex. When the nearest crack is around $140 \mathrm{~mm}$ from the probe

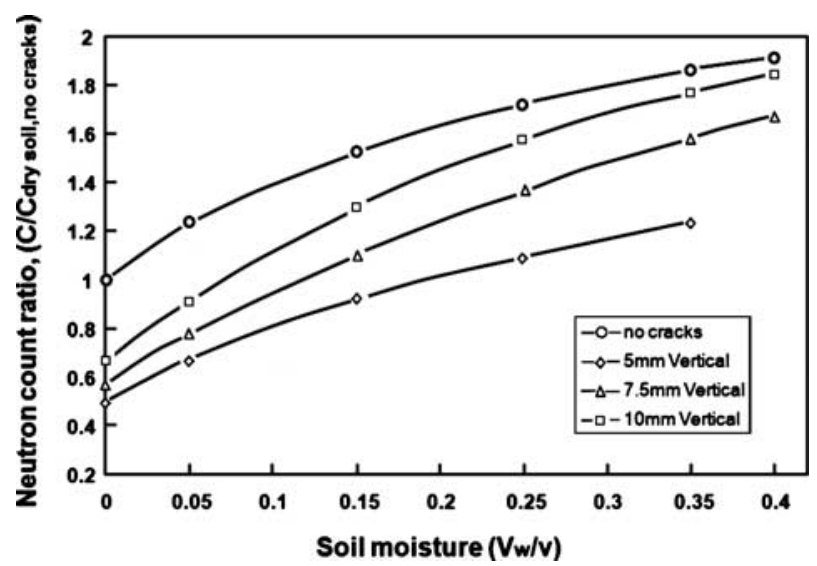

FIG. 8-The impact of air-filled vertical cracks on the neutron count. 


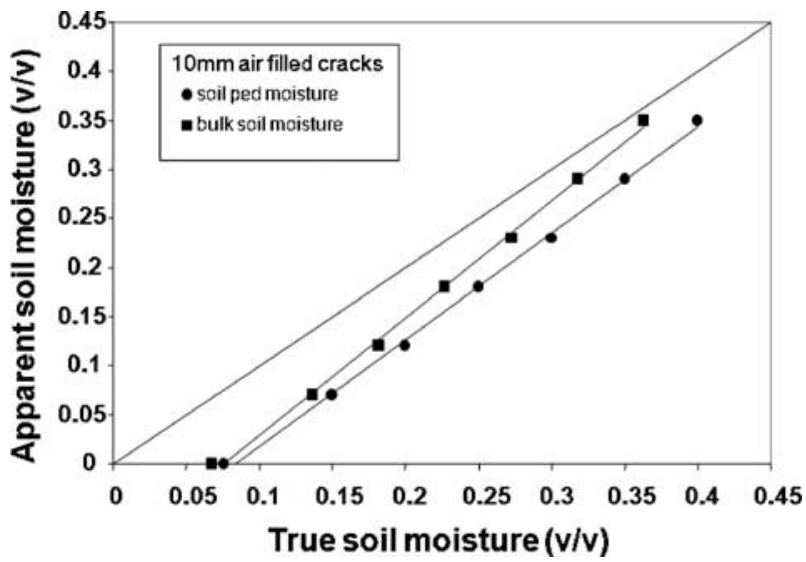

FIG. 9-Effect of $10 \mathrm{~mm}$ air-filled cracking (10\% void fraction) on the prediction of bulk and pedal soil moisture.

axis, the effect of replacing the air in the crack system with water is to return the neutron count to approximately the levels predicted for the case in which no cracks are present. Essentially the probe response is no longer affected by the presence of cracks in the soil. However if the cracks are redistributed so that the nearest crack is located closer to the neutron probe the neutron count is increased above that predicted for the case in which no cracks are present. This would result in the opposite effect to that cited previously for the case of air-filled cracking,: that is, an over prediction of soil moisture levels. In this case the presence of a body of water close to the neutron source is acting to block the passage of neutrons diffusing away from the neutron source and to artificially concentrate the neutron cloud in the region of the sensor, thereby increasing the neutron count.

\section{The Effect of Horizontal Cracking}

Figure 13 shows the influence of horizontal cracks on the response of the neutron probe over a range of soil moistures. In this scenario the neutron probe is surrounded by a series of planar disk-shaped cracks that commence at $30 \mathrm{~mm}$ from the probe axis, extending to 1 $\mathrm{m}$ from the probe axis. Crack spacing has once again been set to produce an overall $10 \%$ void fraction within the cracked soil domain. As observed in the case of vertical cracking, the neutron count is predicted to decrease in the presence of air-filled cracks and increase when cracks are filled with water. The overall effect for horizontal cracking, however, is not as pronounced possibly because the vertical cracks present a greater surface area to the diffus-
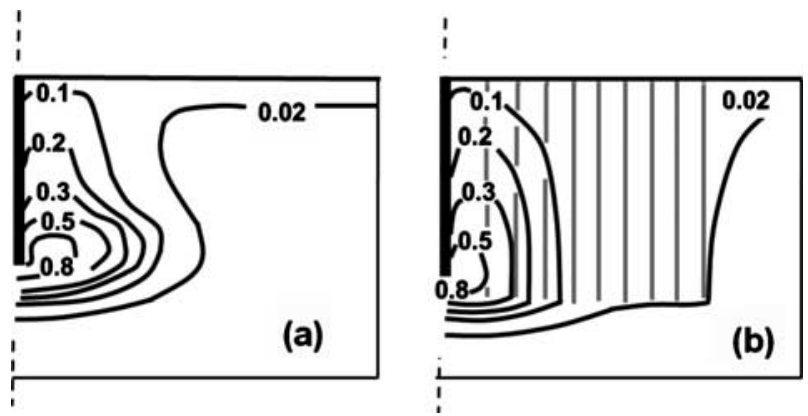

FIG. 10 - Contour plot of the distribution of the thermal neutron cloud. (a) soil with no cracks and (b) soil with $10 \mathrm{~mm}$ cracks (shown in gray), $10 \%$ void fraction. Neutron count contours are normalised to the maximum neutron count.

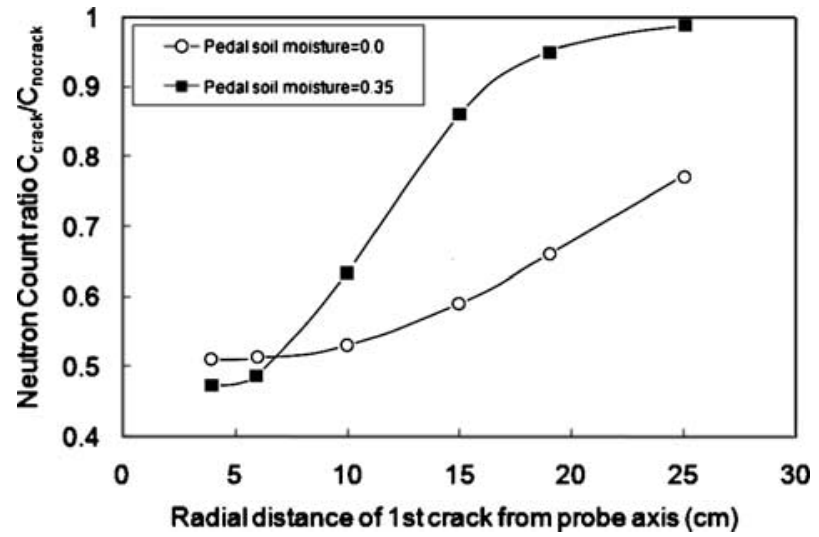

FIG. 11-The effect on the neutron count of proximity of cracking to the probe.

ing neutrons (which are primarily fluxing from the source in a radial direction). It is natural to expect that many of the neutrons would tend to travel in the discoid, uncracked soil domains located between the planar cracks, thereby lessening the impact of the crack network.

In contrast to the previous results, it is also apparent that the discrepancy between cracked and non-cracked readings increases with increasing soil moisture when air-filled cracks are present. A close inspection of Fig. 10 reveals a slight distension of the neutron cloud in the radial direction when no cracks are present (particularly when soil moisture is low). Horizontal air-filled cracks will pull the neutron cloud in the same direction (i.e., radially away from the source/sensor). The overall effect of this type of cracking on the neutron distribution, especially at low soil moisture levels, is therefore not as pronounced as was predicted for the scenarios involving vertical cracking (where the neutron cloud is distended vertically). At higher soil moisture levels, the neutron cloud is more spherical in shape and the extension of the cloud in the radial direction by air-filled horizontal cracks is more pronounced. Consequently, as soil moisture increases, the impact of horizontal cracks will increase, while that of vertical cracking will decrease.

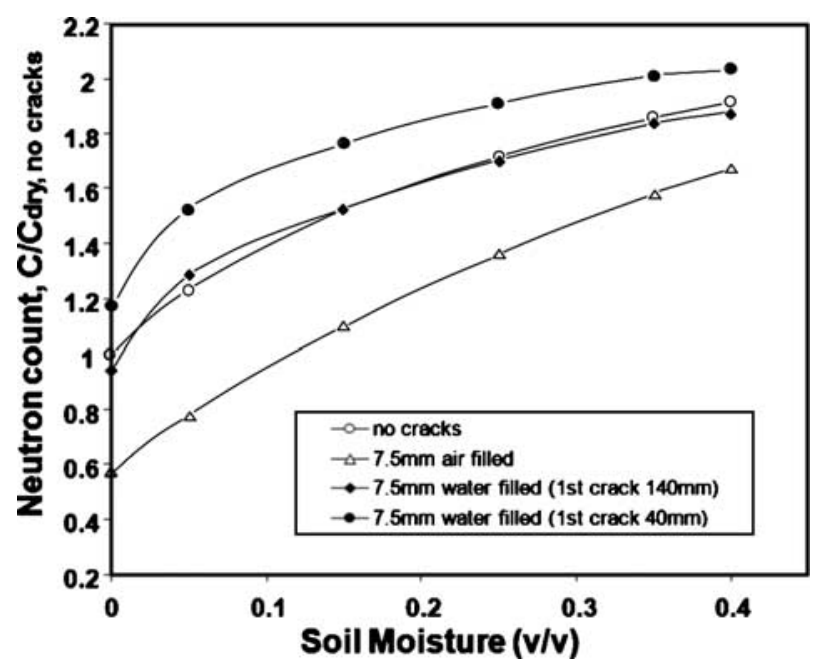

FIG. 12-Effect of water-filled vertical cracks on the neutron count, $7.5 \mathrm{~mm}$ vertical cracks, $10 \%$ void fraction. 


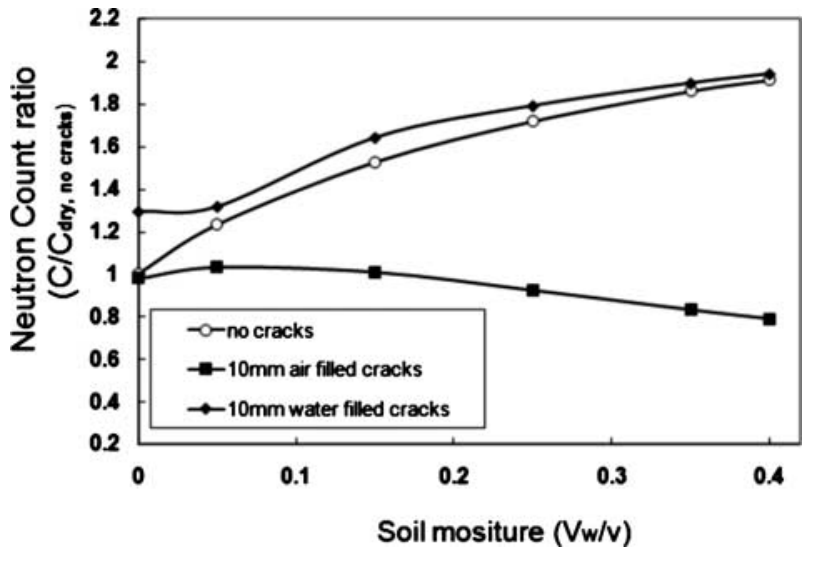

FIG. 13-The impact of water and air-filled horizontal cracking on the neutron count.

\section{Refined Analysis of Moisture Measurement in a Cracked Expansive Soil}

A more refined analysis using the more consistent soil volume change model described above was also performed, which employed realistic crack widths, depths, and spacings, which are a function of water content. In soils such as the Maryland clay, a stable and persistent crack network has been developed over many weather cycles (Fityus and Smith 2004). Cracks open or close depending on the water content in the soil, and their volume and depth are assumed to be proportional to the volume of shrinkage experienced by the soil. Based on observations made at the Maryland site (Moe et al. 2003), regularly distributed cracks at spacings of 8, 14 and $20 \mathrm{~cm}$ were used in this model. For these three cases, the first crack was placed at a distance of 4,7 , and $10 \mathrm{~cm}$, respectively, from the symmetric axis. In each case the access tube was located in the centre of a crack-bounded soil block. The neutron probe was placed in the access tube $100 \mathrm{~cm}$ beneath the ground surface.

It has been observed that cracks start to open at a gravimetric water content $w<w_{c}=36 \%$, becoming wider until they reach a shrinkage limit of $w_{o}=16 \%$. Cracks typically become narrower with depth down to a maximum depth of $120 \mathrm{~cm}$. The depth and the width of cracks used in this analysis were determined based on realistic changes of gravimetric water content occurring with depth. The soil profile was modelled using a piecewise constant variation, consisting of a series of five $24 \mathrm{~cm}$ thick layers in which the water content and hence the crack width are locally constant. For cracks of a given depth, it was assumed that the soil in the layer beneath the crack had a water content of $w_{c}=36 \%$, with the water content in layers above decreasing in increments of $\Delta w=4 \%$. Therefore for cracks to the maximum depth of $120 \mathrm{~cm}$ the water contents range from $w_{o}=16 \%$ in the uppermost layer up to $w=32 \%$ in the fifth layer, with the soil below $120 \mathrm{~cm}$ modelled as $w_{c}=36 \%$.

The volume change in soil can be calculated according to

$$
\frac{\Delta v}{v_{c}}=\frac{\psi G_{s} \Delta w}{\psi G_{s}\left(w_{c}-w_{0}\right)+1+e_{0}}
$$

where:

$v_{c}$ represents the specific volume of soil at $w_{c}=36 \%$ and $\Delta v=$ variation of the specific volume.

The increase in the width of cracks $\Delta d$ is determined from
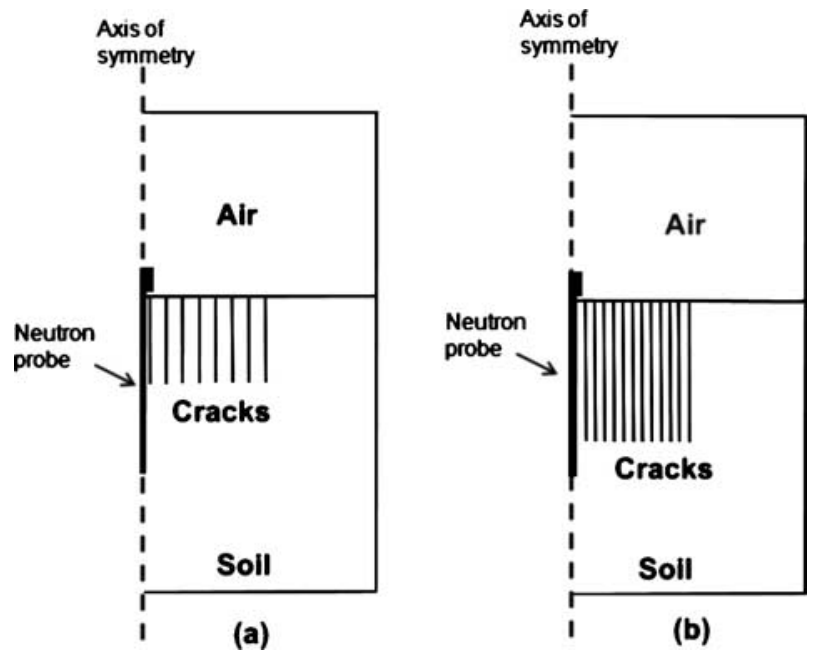

FIG. 14-Sketch of computing model with cracks (a) $72 \mathrm{~cm}$ of depth at $14 \mathrm{~cm}$ spacing for $w=24 \%$ and (b) $120 \mathrm{~cm}$ of depth at $8 \mathrm{~cm}$ spacing for $w=16 \%$.

$$
\Delta d / l=c_{h} \frac{\Delta v}{v_{c}}
$$

where:

$c_{h}=$ factor that represents the fraction of the total shrinkage that acts in each spatial direction, (a value of 1/3 is assumed).

As an illustration of the situations modelled, sketches of the numerical model with crack distributions as described above are presented in Fig. 14 for surface water contents of $w=24 \%$ and $w$ $=16 \%$ and corresponding crack depths of $72 \mathrm{~cm}$ and $120 \mathrm{~cm}$, respectively. The change in the dry density of the uncracked soil peds as a function of water content was also taken into account as described above. It was assumed that the cracks are filled with air.

Numerical results are presented in Fig. 15. It can be seen that the neutron count ratio as a function of the volumetric water content is no longer linear. The $C_{r}-\theta$ relations are strongly curved. Cracks in the soil generally reduce the neutron count, but when the cracks are shallow and the neutron probe is placed at some depth away from the cracks, such an effect is not pronounced. When the cracks reach the depth where the neutron probe is placed, the neutron count ratio is reduced significantly. The crack spacing, as well as the distance

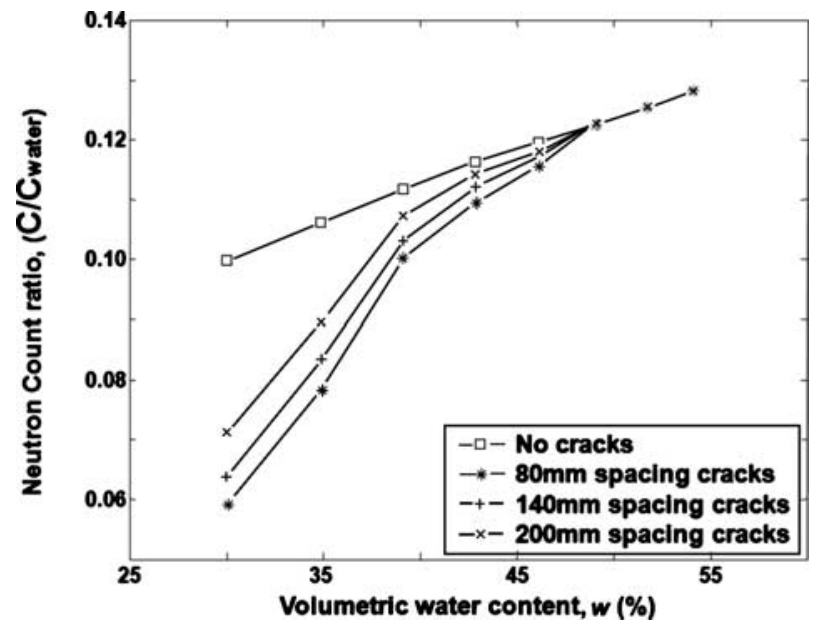

FIG. 15-Neutron count ratio obtained with cracks developed with the variation of water content. 
of the neutron probe from the crack, also affects the neutron counts detected by the sensor. $C_{r}-\theta$ curves of similar shape are obtained for the three computation cases. For the same crack volume, the neutron count ratio $C_{r}$ is lower for finer cracks with denser distribution. These results suggest that with the presence of soil cracking, a linear correlation between neutron counts and soil water content is unlikely to be valid. In such situation, neutron measurement of water content based on a linear calibration may be unreliable.

\section{Conclusions}

The fact that the neutron probe "senses" soil moisture over an extended volume of soil from within a pre-prepared shaft would suggest that its use would confer advantages in the monitoring of soil moisture in expansive clays. The predictions of a 3D finite element model of the neutron probe process however indicate that the presence of cracks in the immediate vicinity of the probe can have a significant influence on the neutron count reported by the probe, and hence the use of such devices in expansive clays must be treated with some caution.

The model results revealed that the neutron count is a function of the orientation of the cracking; whether it is air or water-filled; the proximity of cracking to the neutron probe as well as the width of cracking. Some general conclusions to be drawn from the model predictions include: air-filled cracks tend to suppress the neutron count (leading to an under-prediction of soil moisture); water-filled cracks produce a (lesser) increase neutron count (resulting in an over prediction of the soil moisture content); and vertical cracks appear to have a greater effect on the neutron count than horizontal cracks. The exact magnitude of the error that is introduced by the presence of cracks however would be difficult to predict as it arises from a complex interaction between the neutron cloud and the presence of preferential transport pathways (air-filled cracks) or collisional/absorptive pathways (water-filled cracks). To account for such a deviation would require knowledge of the location and spatial distribution of the crack network that is unlikely to be available in a normal field situation.

It has also been found from the numerical modelling that the density change in an expansive soil affects the value of calibration parameter but does not change the linear relation between neutron count and volumetric water content. When the development of cracks is taken into account, however, a nonlinear relation between neutron count and volumetric water content is obtained, and this consequently has a significant effect on the accurate measurement of water content in soil which is usually based on a linear calibration.

\section{References}

Amoozegar, A., Martin, K. C., and Hoover, M. T., 1989, “Effect of Access Hole Properties on Soil Water Content Determination by Neutron Thermalisation," Soil Sci. Soc. Am. J., Vol. 53, pp. 330335.

Corbeels, M., Hartmann, R., Hofman, G., and Van Cleemput, O., 1999, "Field Calibration of a Neutron Moisture Meter in Vertisols," Soil Sci. Soc. Am. J., Vol. 63, pp. 11-18.
Elder, A. N. and Rasmussen, T. C., 1994, "Neutron Probe Calibration in Unsaturated Tuff," Soil Sci. Soc. Am. J., Vol. 58(5), pp. 1301-1307.

Fityus, S. and Buzzi, O., 2009, "The Place of Expansive Clays in the Framework of Unsaturated Soil Mechanics," Appl. Clay Sci., Vol. 43, pp. 150-155.

Fityus, S. G. and Smith, D. W., 2004, "The Development of a Residual Soil Profile from a Mudstone in a Temperate Climate," Eng. Geol. (Amsterdam), Vol. 74(1-2), pp. 39-56.

Fityus, S. G., Smith, D. W., and Allman, M. A., 2004, "Expansive Soil Test Site near Newcastle," J. Geotech. Geoenviron. Eng., Vol. 130(7), pp. 686-695.

Greacen, E. L. and Hignett, C. T., 1979, "Sources of Bias in the Field Calibration of a Neutron Meter," Austral. J. Soil Res., Vol. 17, pp. 405-415.

Greacen, E. L. and Schrale, G., 1976, "The Effect of Bulk Density on Neutron Meter Calibration," Austral. J. Soil Res., Vol. 14(2), pp. 159-169.

Grismer, M. E., Bali, K. M., and Robinson, F. E., 1995, "FieldScale Neutron Probe Calibration and Variance Analysis for Clay Soil,” J. Irrig. Drain. Eng., Vol. 121(5), pp. 354-362.

Haahr, V. and Ølgaard, P. L., 1965, “Comparative Experimental and Theoretical Investigations of the Neutronic Method for Measuring the Water Content in Soil," Symp. on Use of Isotope and Radiation in Soil-Plant Nutrition Studies, Ankara, June 28-July 2, IAEA, Vienna, pp. 129-147.

Haines, W. B., 1923, "The Volume Changes Associated with Variations of Water Content in Soil," J. Agric. Sci. Vol. 13, pp. 296310.

Hanafy, E. A. D. E., 1991, "Swelling/Shrinkage Characteristic Curve of Desiccated Expansive Clays," Geotech. Test. J., Vol. 14(2), pp. 206-211.

Huang, W. and Fityus, S. G., 2008, "Numerical Study of Neutron Probe Measurement of Water Content in Expansive Soils," Proceedings of the 12th International Conference of International Association for Computer Methods and Advances in Geomechanics (IACMAG) (CD-ROM), Goa, India, Oct. 1-6.

Iliffe, C. E., 1982, An Introduction to Nuclear Reactor Physics, Manchester University Press, England.

Jarvis, N. J. and Leeds-Harrison, P. B., 1987, "Some Problems Associated with the Use of the Neutron Probe in Swelling/ Shrinking Clay Soils," J. Soil Sci., Vol. 38, pp. 149-156.

Jayawardane, N. S., Meyer, W. S., and Barrs, H. D., 1984, "Moisture Measurement in a Swelling Clay Soil Using Neutron Moisture Meters," Austral. J. Soil Res., Vol. 22, pp. 109-117.

Kodikara, J., Barbour, S. L., and Fredlund, D. G., 2002, "Structure Development in Surficial Heavy Clay Soils: A Synthesis of Mechanisms," Austral. Geomech., Vol. 37(1), pp. 25-40.

Li, J., Smith, D. W., and Fityus, S. G., 2003b, "The Effect of a Gap Between the Access Tube and the Soil During Neutron Probe Measurements," Austral. J. Soil Res., Vol. 41(1), pp. 151-164.

Li, J., Smith, D. W., Fityus, S. G., and Sheng, D., 2003a, "Numerical Analysis of Neutron Moisture Probe Measurement," Int. J. Geomech., Vol. 3(1), pp. 11-20.

Moe, H., Fityus, S. G., and Smith, D. W., 2003, "Study of a Cracking Network in a Residual Clay Soil," Proceedings of UNSATASIA 2003: Second Asian Unsaturated Soils Conference, Osaka, Japan, April 15-17, D. Karube, A. Iizuka, S. Kato, K. Kawai, and K. Tateyama, Eds., Organizing Committee of UNSATASIA 2003, Osaka, Japan, pp. 149-154.

Ølgaard, P. L., 1965, “On the Theory of the Neutronic Method for Measuring the Water Content in Soil," Risö Report No. 97, Dan- 
ish Atomic Energy Commission Research Establishment, Roskilde, Denmark.

Ritchie, A. I. M. and Wilson, D. J., 1984, "Investigation of the Response of a Neutron Moisture Meter Using a Multigroup, TwoDimensional Diffusion Theory Code," Report No. AAEC/E579, Australian Atomic Energy Commission Research Establishment.

Tripathy, S., Subba Rao, K. S., and Fredlund, D. G., 2002, "Water
Content-Void Ratio Swell-Shrink Paths of Compacted Expansive Soils," Can. Geotech. J., Vol. 39, pp. 938-959.

Wells, T. and Fityus, S., 2007, "The Influence of Desiccation Cracking on Volumetric Moisture Contents Measured by the Neutron Probe," Proc. Third Asian Conf. on Unsaturated Soils, Nanjing, China, April 2007, Z. Yin, J. Yuan, and A. F. Chiu, Eds., Science Press, Beijing, China, pp. 593-598. 